\title{
Surface Water Quality Impacts of Conservation Tillage Practices on Burley Tobacco Production Systems in Southwest Virginia
}

\author{
Brian L. Benham • Megan K. Laird • Blake B. Ross • \\ David H. Vaughan • Danny R. Peek
}

Published online: 24 October 2006

(C) Springer Science + Business Media B.V. 2006

The author order should be Benham, Laird, Ross, Vaughan, and Peek.

The online version of the original article can be found at: http://dx.doi.org/10.1007/s11270-006-9221-z

B. L. Benham $(\bowtie) \cdot$ M. K. Laird $\cdot$ B. B. Ross $\cdot$ D. H. Vaughan

Biological Systems Engineering Department,

Virginia Tech, 209 Seitz Hall,

Blacksburg, VA 24061, USA

e-mail: benham@vt.edu

D. R. Peek

Southern Piedmont Ag Research and Extension Center,

Virginia Tech, Blacksburg, VA, USA 